\title{
Extension of modular functions and measures
}

\author{
Hans Weber $^{1}$ (D)
}

Received: 1 July 2017 / Accepted: 30 September 2017 / Published online: 13 October 2017

(C) Fondazione Annali di Matematica Pura ed Applicata and Springer-Verlag GmbH Germany 2017

\begin{abstract}
We prove extension theorems for group-valued modular functions defined on orthomodular lattices or modular complemented lattices and for modular measures defined on (pseudo-)D-lattices generalizing results of Riečan (Mat Časopis Sloven Akad Vied 19:4449, 1969; 20:239-244, 1970; Comment Math Univ Carolin 20:309-316, 1979), Avallone and De Simone (Ital J Pure Appl Math 9:109-122, 2001) and Avallone et al. (Bollettino UMI 9-B(8):423-444, 2006; Math Slovaca 66:421-438, 2016). As basic tool, we first prove an extension theorem for lattice uniformities. This also yields as immediate consequence a result on the extension of modular functions on arbitrary lattices similar to that of Fox and Morales (Fundam Math 78:99-106, 1973) and Kranz (Fundam Math 91:171-178, 1976).
\end{abstract}

Keywords Modular function · Measure · Extension · Lattice uniformity · Orthomodular lattice $\cdot$ Modular complemented lattice $\cdot$ D-lattice

Mathematics Subject Classification 28E99 $\cdot 28 \mathrm{~B} 10 \cdot 06 \mathrm{~B} 30 \cdot 06 \mathrm{C} 15$

\section{Introduction}

Carathéodory's theorem on the extension of a $\sigma$-additive positive measure from an algebra of sets on the generated $\sigma$-algebra has been generalized in different directions. Sion [16] was the first who generalized this theorem for group-valued measures. Riečan [12-14] proved extension theorems for "measures" defined on a more general domain, namely on orthomodular lattices or modular complemented lattices. Avallone and De Simone have given in [2] another proof (using a topological approach) of Riečan's extension theorem for positive

1 The "measures" considered in these articles of Riečan are exactly $\sigma$-order continuous modular functions $\mu$ (as defined in Sect. 2) satisfying $\mu(0)=0$. To see this in case [14] use [15, Lemma 1].

Hans Weber

hans.weber@uniud.it

1 Dipartimento di Science Matematiche, Informatiche e Fisiche, Università degli Studi di Udine, Via delle Scienze 206, 33100 Udine, Italy 
measures on orthomodular lattices. As a consequence, they obtained an extension theorem for measures which can be "controlled" by a family of positive measures; in particular, this implies (employing a control measure theorem) an extension theorem for measures defined on an orthomodular lattice with values in a locally convex space. These results of [2] are generalized in [3] for modular measures on D-lattices ${ }^{2}$ and recently for modular measures on pseudo-D-lattices [4].

The main aim of this article is to prove these extension theorems for measures (defined on orthomodular lattices, more general on D-lattices, or on modular complemented lattices) with values in topological groups (in particular in not necessarily locally convex topological linear spaces). For that, we first prove extension theorems for lattice uniformities; the measure extension one gets then easily as continuous extension with respect to a suitable lattice uniformity. With this procedure, the problem to extend measures (or modular functions) can completely be reduced to the problem of extension of lattice uniformities. In particular, this shows that here the structure of the range space plays an unimportant role. More precisely, let $S$ be a sublattice of a $\sigma$-continuous $\sigma$-complete lattice $L$ and $\sigma(S)$ be the $\sigma$-order closed sublattice generated by $S$. We first give a necessary and sufficient condition for extending an exhaustive $\sigma$-order continuous ( $\sigma$-o.c. for short) lattice uniformity $u$ on $S$ to a $\sigma$-o.c. lattice uniformity $\bar{u}$ on $\sigma(S)$ (Corollary 4.6). This condition is satisfied, e.g. if $L$ is an orthomodular lattice and $S$ a sub-orthomodular lattice of $L$ (Corollary 4.9), or if $L$ is a modular complemented lattice and $S$ a sublattice of $L$ containing all complements of any $x \in S$ (Corollary 4.8). This is applied in Sect. 5 where $u$ is the $\mu$-uniformity of an exhaustive $\sigma$-o.c. group-valued modular function $\mu: S \rightarrow G$. Since $S$ is dense in $(\sigma(S), \bar{u}), \mu$ can be extended by continuity to a $\sigma$-o.c. modular function on $\sigma(S)$. This yields always an extension theorem for modular functions when $L$ is an orthomodular lattice (Theorem 5.3) or a modular complemented lattice (Theorem 5.2). When $L$ is an arbitrary lattice, it yields a necessary and sufficient condition for extending an exhaustive $\sigma$-o.c. modular function $\mu: S \rightarrow G$ to a $\sigma$-o.c. modular function on $\sigma(S)$ (Theorem 5.1); (2) $\Leftrightarrow(3)$ of 5.1 is essentially equivalent to the result of Kranz [11] who generalized a result of Fox and Morales [10] replacing lattices of sets by abstract lattices.

The case that $L$ is a D-lattice we treat separately in Sect. 6.1. We first show that any exhaustive $\sigma$-o.c. D-lattice uniformity on a D-sublattice $S$ of a $\sigma$-continuous $\sigma$-complete D-lattice $L$ can be extended to a $\sigma$-o.c. D-lattice uniformity on $\sigma(S)$ (Corollary 6.6). An immediate consequence (Corollary 6.7) is an extension theorem for group-valued modular measures from $S$ to $\sigma(S)$ which generalizes results of Avallone et al. [3]. The system $\sigma(S)$ turns out to be a $\sigma$-complete D-sublattice of $L$ (see Theorem 6.4 or [3, Theorem 3.4]). In Sect. 6.2, we briefly explain how to transfer the results of Sect. 6.1 in the setting of pseudoD-lattices.

Throughout, let $L$ be a lattice and $G=(G,+, \tau)$ a commutative Hausdorff topological group.

\section{Preliminaries}

Let $\left(x_{n}\right)$ be a sequence in $L$ and $x \in L$. We write $x_{n} \uparrow$ if $\left(x_{n}\right)$ is increasing, and $x_{n} \uparrow x$ if $\left(x_{n}\right)$ is increasing and $x=\sup _{n \in \mathbb{N}} x_{n}$. Analogously, $x_{n} \downarrow$ and $x_{n} \downarrow x$ are defined.

${ }^{2}$ D-lattices are a common generalization of orthomodular lattices and MV-algebras. For the significance of this structure, see [7]. 
For $A \subseteq L$ let

$$
\begin{aligned}
& A_{\sigma}=\left\{x \in L: \exists\left(x_{n}\right) \in A^{\mathbb{N}} \quad x_{n} \uparrow x\right\}, \\
& A_{\delta}=\left\{x \in L: \exists\left(x_{n}\right) \in A^{\mathbb{N}} \quad x_{n} \downarrow x\right\} .
\end{aligned}
$$

$L$ is called $\sigma$-continuous if $x_{n} \uparrow x$ implies $x_{n} \wedge y \uparrow x \wedge y$ and $x_{n} \downarrow x$ implies $x_{n} \vee y \downarrow x \vee y$ for any sequence $\left(x_{n}\right)$ in $L$ and $x, y \in L$. If $L$ is $\sigma$-continuous and $A$ a sublattice of $L$, then $A_{\sigma}$ and $A_{\delta}$ are also sublattices of $L$.

For $A \subseteq L$, we denote by $\sigma(A)$ the smallest subset $M$ of $L$ containing $A$ with $M=M_{\sigma}=$ $M_{\delta}$.

Proposition 2.1 If $L$ is $\sigma$-continuous and $A$ a sublattice of $L$, then also $\sigma(A)$ is a sublattice of $L$.

Proof Let $\mathfrak{A}$ be the set of all subsets $T$ of $\sigma(S)$ containing $A$ such that $T \vee T \subseteq \sigma(S)$ and $T \wedge T \subseteq \sigma(S)$. Then $S \in \mathfrak{A}$. Let $M$ be a maximal element of $\mathfrak{A}$. Obviously, $M_{\sigma} \vee M_{\sigma} \subseteq$ $(M \vee M)_{\sigma} \subseteq \sigma(S)_{\sigma}=\sigma(S)$. Moreover, since $L$ is $\sigma$-continuous, $M_{\sigma} \wedge M_{\sigma} \subseteq(M \wedge M)_{\sigma} \subseteq$ $\sigma(S)_{\sigma}=\sigma(S)$. Hence $M_{\sigma} \in \mathfrak{A}$, and thus $M_{\sigma}=M$ by the maximality of $M$. Dually, we have $M_{\delta}=M$. Therefore $\sigma(M)=M$ and finally $M=\sigma(S)$, since $S \subseteq M \subseteq \sigma(S)$.

Let $u$ be a lattice uniformity on $L$, i.e. a uniformity on $L$ making the lattice operations $\wedge$ and $\vee$ uniformly continuous. We call $u$ exhausive if every monotone sequence (equivalently, every monotone net) in $L$ is $u$-Cauchy (cf. [17, Definition 1.2.5], [18, Proposition 6.1]). A subset $A \subseteq L$ is lower dense (upper dense) in ( $L, u)$ if for every $x \in L$ and $U \in u$ there exists an element $a \in A$ with ( $a, x) \in U$ and $a \leq x$ (respectively, $a \geq x$ ).

Let $D(L)$ be the system of all semimetrics $d: L^{2} \rightarrow[0,+\infty[$ satisfying $d(x \vee z, y \vee z) \leq$ $2 d(x, y)$ and $d(x \wedge z, y \wedge z) \leq 2 d(x, y)$ for all $x, y, z \in L$. Obviously, the uniformity induced by a nonempty subset of $D(L)$ is a lattice uniformity. Vice versa, every lattice uniformity on $L$ is generated by a nonempty subset of $D(L)$, see [19, Corollay 1.4].

A semimetric $d \in D(L)$ is called exhaustive if so is the $d$-uniformity.

\section{Lemma 2.2 Let $d \in D(L)$.}

(a) $d\left(\sup _{i \leq n} x_{i}, \sup _{i \leq n} y_{i}\right) \leq \sum_{i=1}^{n} 2^{i} d\left(x_{i}, y_{i}\right)$ and $d\left(\inf _{i \leq n} x_{i}, \inf _{i \leq n} y_{i}\right) \leq \sum_{i=1}^{n} 2^{i} d\left(x_{i}\right.$, $y_{i}$ ) whenever $x_{i}, y_{i} \in L$ for $i=1, \ldots, n$.

(b) If $x, y, z \in L$ with $x \leq y \leq z$, then $d(x, y) \leq 2 d(x, z)$ and $d(y, z) \leq 2 d(x, z)$.

Proof (a) For the first inequality, one verifies by induction

$$
d\left(\sup _{i \leq m} x_{i}, \sup _{i \leq m} y_{i}\right) \leq \sum_{i=1}^{m-1} 2^{i} d\left(x_{i}, y_{i}\right)+2^{m-1} d\left(x_{m}, y_{m}\right) \text { for } 1 \leq m \leq n .
$$

The second inequality then follows by duality.

(b) $d(x, y)=d(x \wedge y, z \wedge y) \leq 2 d(x, z)$ and $d(y, z)=d(x \vee y, z \vee y) \leq 2 d(x, z)$.

Lemma 2.3 Let $d \in D(L)$ and $K$ be a lower-dense sublattice of $(L, d)$. Let $\left(x_{n}\right)$ be a decreasing sequence in $L$ and $\varepsilon>0$. Then there is a decreasing sequence $\left(z_{n}\right)$ in $K$ such that $z_{n} \leq x_{n}$ and $d\left(x_{n}, z_{n}\right) \leq \varepsilon$ for all $n \in \mathbb{N}$. 
Proof Choose $y_{n} \in K$ such that $y_{n} \leq x_{n}$ and $d\left(y_{n}, x_{n}\right) \leq 4^{-n} \varepsilon$ for $n \in \mathbb{N}$. Then $z_{n}:=$ $\inf _{i \leq n} y_{i}$ defines a decreasing sequence in $K$ and

$$
d\left(x_{n}, z_{n}\right)=d\left(\inf _{i \leq n} x_{i}, \inf _{i \leq n} y_{i}\right) \leq \sum_{i=1}^{n} 2^{i} d\left(x_{i}, y_{i}\right) \leq \sum_{i=1}^{n} 2^{i} 4^{-i} \varepsilon \leq \varepsilon .
$$

Let $\mu: L \rightarrow G$ be a modular function, i.e. $\mu(x \vee y)+\mu(x \wedge y)=\mu(x)+\mu(y)$ for all $x, y \in L$. Then the sets

$$
\left\{(a, b) \in L^{2}: \mu(x)-\mu(y) \in U \text { whenever } x, y \in[a \wedge b, a \vee b]\right\} \text { where } U
$$
is a 0 -neighbourhood in $G$

form a base of the weakest lattice uniformity making $\mu$ uniformly continuous (see [9]); this uniformity is called the $\mu$-uniformity. The proof of the next proposition is contained in [9].

Proposition 2.4 Let $\mu: L \rightarrow G$ be a modular function and $\left(||_{\gamma}\right)_{\gamma \in \Gamma}$ be a system of bounded group seminorms generating the topology $\tau$ of $G$. Then

$$
d_{\gamma}(a, b):=\sup \left\{|\mu(x)-\mu(y)|_{\gamma}: x, y \in[a \wedge b, a \vee b], x \leq y\right\}, \quad \gamma \in \Gamma,
$$

defines a system of semimetrics on $L$ which generates the $\mu$-uniformity. Moreover,

$d_{\gamma}(x \vee z, y \vee z) \leq d_{\gamma}(x, y)$ and $d_{\gamma}(x \wedge z, y \wedge z) \leq d_{\gamma}(x, y)$ for all $x, y, z \in L$ and $\gamma \in \Gamma$, in particular $d_{\gamma} \in D(L)$ for $\gamma \in \Gamma$.

A modular function $\mu: L \rightarrow G$ is exhaustive if $\left(\mu\left(x_{n}\right)\right)_{n \in \mathbb{N}}$ is Cauchy for every monotone sequence $\left(x_{n}\right)$ in $L$. By $[21,3.6] \mu$ is exhaustive iff the $\mu$-uniformity is exhaustive.

Let $u$ be a lattice uniformity on a sublattice $M$ of $L$. We call $u$ lower $\sigma$-order continuous (upper $\sigma$-order continuous) if $x_{n} \uparrow x$ (respectively $\left.x_{n} \downarrow x\right)^{3}$ implies $x_{n} \rightarrow x(u)$ for any sequence $\left(x_{n}\right)$ in $M$ and $x \in M . u$ is $\sigma$-order continuous if $u$ is both, lower $\sigma$-o.c. and upper $\sigma$-o.c. A semimetric $d \in D(M)$ is, respectively, $\sigma$-o.c., lower $\sigma$-o.c., upper $\sigma$-o.c. if the $d$-uniformity is so.

A modular function $\mu: M \rightarrow G$ is lower $\sigma$-o.c. (upper $\sigma$-o.c.) if $x_{n} \uparrow x$ (respectively $\left.x_{n} \downarrow x\right)$ implies $\mu\left(x_{n}\right) \rightarrow \mu(x)(\tau)$ for any sequence $\left(x_{n}\right)$ in $M$ and $x \in M$. If $\mu$ is both, lower $\sigma$-o.c. and upper $\sigma$-o.c., then $\mu$ is called $\sigma$-o.c. .

More general, one can define for an infinite cardinal number $\kappa$ (lower, upper) $\kappa$-order continuity replacing in the definition sequences by nets $\left(x_{\gamma}\right)_{\gamma \in \Gamma}$ in $M$ with $|\Gamma| \leq \kappa$. The proof of [21, Proposition 3.5] shows that for a modular function $\mu: M \rightarrow G$ the $\mu$-uniformity is lower $\kappa$-o.c. (upper $\kappa$-o.c.) iff $\mu\left(x_{\gamma}\right) \rightarrow \mu(x)$ whenever $x \in M$ and $\left(x_{\gamma}\right)_{\gamma \in \Gamma}$ is a net in $M$ such that $|\Gamma| \leq \kappa$ and $x_{\gamma} \uparrow x$ (respectively $x_{\gamma} \downarrow x$ ). Here we are interested only in the case that $\kappa$ is countable:

Proposition 2.5 Let $\mu: M \rightarrow G$ be a modular function defined on a sublattice of $L$. Then $\mu$ is lower $\sigma$-o.c. (upper $\sigma$-o.c.) iff the $\mu$-uniformity is lower $\sigma$-o.c. (respectively, upper $\sigma$-o.c.).

Lemma 2.6 Let $L$ be $\sigma$-complete, $M$ and $K$ sublattices of $L$ and $d \in D(M)$.

(a) Suppose that $K_{\delta} \subseteq M$ and $K$ is lower dense in $(M, d)$. If $d\left(y_{n}, y\right) \rightarrow 0(n \rightarrow+\infty)$ whenever $\left(y_{n}\right)$ is a decreasing sequence in $K$ with $y_{n} \downarrow y \in L$, then $d$ is upper $\sigma$-o.c.

3 In this article the supremum or infimum of a subset of a sublattice $M$ of $L$ is always taken in $L$ (not in $M$ ). 
(b) Suppose that $K_{\sigma} \subseteq M$ and $K$ is upper-dense in $(M, d)$. If $d\left(y_{n}, y\right) \rightarrow 0(n \rightarrow+\infty)$ whenever $\left(y_{n}\right)$ is a increasing sequence in $K$ with $y_{n} \uparrow y \in L$, then $d$ is lower $\sigma$-o.c.

Proof We only proof (a); (b) then follows by duality. Let $x_{n}, x \in M$ with $x_{n} \downarrow x$ and $\varepsilon>0$. Choose $z \in K$ such that $z \leq x$ and $d(z, x) \leq \varepsilon$, and a decreasing sequence $\left(z_{n}\right)$ in $K$ such that $z_{n} \leq x_{n}$ and $d\left(z_{n}, x_{n}\right) \leq \varepsilon$ for $n \in \mathbb{N}$ (see Lemma 2.3). Let $y_{n}:=z_{n} \vee z$ and $y:=\inf _{n \in \mathbb{N}} y_{n} \in K_{\delta} \subseteq M$. Then $d\left(x_{n}, y_{n}\right)=d\left(x_{n} \vee z, z_{n} \vee z\right) \leq 2 d\left(x_{n}, z_{n}\right) \leq 2 \varepsilon$ and $d(y, x) \leq 2 d(z, x) \leq 2 \varepsilon$ by Lemma 2.2(b). It follows $d\left(x_{n}, x\right) \leq d\left(x_{n}, y_{n}\right)+d\left(y_{n}, y\right)+$ $d(y, x) \leq 4 \varepsilon+d\left(y_{n}, y\right)$. Since $d\left(y_{n}, y\right) \rightarrow 0(n \rightarrow+\infty)$, we have $d\left(x_{n}, x\right) \leq 5 \varepsilon$ eventually.

\section{A uniqueness theorem}

We are interested in extending a lattice uniformity (respectively, a modular function) on $S$ to a $\sigma$-o.c. lattice uniformity (a $\sigma$-o.c. modular function) on $\sigma(S)$. Hereby, the uniqueness part can be proved very easily and without any additional assumption.

Theorem 3.1 Let $S$ be a sublattice of $L$.

(a) If $w$ a $\sigma$-o.c. lattice uniformity on $\sigma(S)$, then $S$ is dense in $(\sigma(S), w)$.

(b) If $u$ and $v$ are $\sigma$-o.c. lattice uniformities on $\sigma(S)$ which induce on $S$ the same uniformity (in symbols $u_{\mid S}=v_{\mid S}$ ), then $u=v$.

(c) If $d_{1}$ and $d_{2}$ are $\sigma$-o.c. semimetrics belonging to $D(\sigma(S))$ which coincide on $S$, then $d_{1}=d_{2}$.

(d) If $\mu, v: \sigma(S) \rightarrow G$ are $\sigma$-o.c. modular functions such that $\mu_{\mid S}=v_{\mid S}$, then $\mu=v$.

Proof (a) Let $C:=\bar{S}$ be the closure of $S$ in $(\sigma(S), w)$. Since $w$ is $\sigma$-o.c., $C \ni x_{n} \uparrow x \in L$ or $C \ni x_{n} \downarrow x \in L$ implies $x \in \sigma(S)$ and $x_{n} \rightarrow x(w)$, hence $x \in \bar{C}=C$, i.e. $C_{\sigma}=C=C_{\delta}$. Thus $C=\sigma(C) \supseteq \sigma(S)$.

(b) Since $w:=u \vee v$ is $\sigma$-o.c., $S$ is dense in $(\sigma(S), w)$ by (a). The assertion now follows from [21, 3.8]. We repeat the proof: since $u \subseteq w$, the closures $\bar{U}^{w \times w}$ of $U \in u_{\mid S}$ taken in $(\sigma(S), w) \times(\sigma(S), w)$ form a base of $u$. Since $u_{\mid S}=v_{\mid S}$, these sets also form a base of $v$. Hence $u=v$.

(c) Let $A:=\left\{x \in \sigma(S): d_{1}(x)=d_{2}(x)\right\}$. Then $S \subseteq A=A_{\sigma}=A_{\delta}$. Hence $A=\sigma(S)$.

(d) In the proof of (c), replace $d_{i}$ by $\mu_{i}$.

\section{Extension of lattice uniformities}

In this section, let $L$ be a $\sigma$-complete $\sigma$-continuous lattice and $S$ a sublattice of $L$.

We are interested in extending a lattice uniformity from $S$ to a $\sigma$-o.c. uniformity on $\sigma(S)$ (see Theorem 4.6 and its corollaries). This is then applied in the next section to obtain extension theorems for modular functions.

Beginning with semimetrics $d \in D(S)$, we extend $d$, in the first step, from $S$ to $S_{\sigma}$.

Proposition 4.1 Let $d \in D(S)$ be exhaustive and lower $\sigma$-o.c.

(a) Then there is a unique lower $\sigma$-order continuous $d_{\sigma} \in D\left(S_{\sigma}\right)$ extending $d$. Moreover, $S$ is lower dense in $\left(S_{\sigma}, d_{\sigma}\right)$, and $d_{\sigma}$ is exhaustive. 
(b) If $K$ is a lower-dense subset of $(S, d)$, then $K$ is lower dense in $\left(S_{\sigma}, d_{\sigma}\right)$, too.

(c) If $K$ is a upper-dense subset of $(S, d)$, then $K_{\sigma}$ is upper dense in $\left(S_{\sigma}, d_{\sigma}\right)$.

Proof (a) Uniqueness: let $\bar{d} \in D\left(S_{\sigma}\right)$ be a lower $\sigma$-o.c. extension of $d$ and $x, y \in S_{\sigma}$. Let $x_{n}, y_{n} \in S$ with $x_{n} \uparrow x$ and $y_{n} \uparrow y$. Then $x_{n} \rightarrow x(\bar{d})$ and $y_{n} \rightarrow y(\bar{d})$, hence $\bar{d}(x, y)=\lim \bar{d}\left(x_{n}, y_{n}\right)=\lim d\left(x_{n}, y_{n}\right)$.

Existence: let $x, y \in S_{\sigma}$ and $x_{n}, y_{n} \in S$ with $x_{n} \uparrow x$ and $y_{n} \uparrow y$. We first show that we can define $d_{\sigma}$ by $d_{\sigma}(x, y):=\lim d\left(x_{n}, y_{n}\right)$. Since $\left(x_{n}\right)$ and $\left(y_{n}\right)$ are Cauchy sequences, the $\lim d\left(x_{n}, y_{n}\right)$ exists. Next we have to show that this limit does not depend on the choice of $\left(x_{n}\right)$ and $\left(y_{n}\right)$. For that, it is enough to show that $d\left(x_{n}, x_{n}^{*}\right) \rightarrow 0$ if $S \ni x_{n}^{*} \uparrow x$ : let $\varepsilon>0$ and $n_{0} \in \mathbb{N}$ such that $d\left(x_{i}, x_{j}\right) \leq \varepsilon$ and $d\left(x_{i}^{*}, x_{j}^{*}\right) \leq \varepsilon$ for $i, j \geq n_{0}$. We show that $d\left(x_{n}, x_{n}^{*}\right) \leq 6 \varepsilon$ for any $n \geq n_{0}$. Fix $n \geq n_{0}$. Since $L$ is $\sigma$-continuous, $x_{j} \wedge x_{n}^{*} \uparrow x \wedge x_{n}^{*}=x_{n}^{*}$ (for $j \rightarrow+\infty)$. Therefore $x_{j} \wedge x_{n}^{*} \rightarrow x_{n}^{*}(d)$. Let $m \geq n$ with $d\left(x_{m} \wedge x_{n}^{*}, x_{n}^{*}\right) \leq \varepsilon$. Then

$d\left(x_{n} \wedge x_{n}^{*}, x_{n}^{*}\right) \leq d\left(x_{n} \wedge x_{n}^{*}, x_{m} \wedge x_{n}^{*}\right)+d\left(x_{m} \wedge x_{n}^{*}, x_{n}^{*}\right) \leq 2 d\left(x_{n}, x_{m}\right)+\varepsilon \leq 2 \varepsilon+\varepsilon=3 \varepsilon$.

Thus we also have $d\left(x_{n} \wedge x_{n}^{*}, x_{n}\right) \leq 3 \varepsilon$ and finally $d\left(x_{n}, x_{n}^{*}\right) \leq d\left(x_{n}, x_{n} \wedge x_{n}^{*}\right)+d\left(x_{n} \wedge\right.$ $\left.x_{n}^{*}, x_{n}^{*}\right) \leq 6 \varepsilon$.

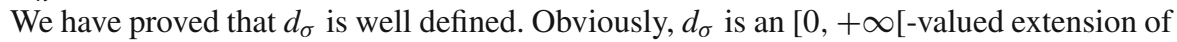
$d$ and $d_{\sigma}$ is symmetric. To verify the triangle inequality, let $x, y, z \in S_{\sigma}$. Let $x_{n}, y_{n} \in S$ as above and $z_{n} \in S$ with $z_{n} \uparrow z$. Then

$$
d_{\sigma}(x, y)=\lim d\left(x_{n}, y_{n}\right) \leq \lim \left(d\left(x_{n}, z_{n}\right)+d\left(z_{n}, y_{n}\right)\right)=d_{\sigma}(x, z)+d_{\sigma}(z, y) .
$$

Moreover, by the $\sigma$-continuity of $L$ we have $x_{n} \wedge z_{n} \uparrow x \wedge z$ and $y_{n} \wedge z_{n} \uparrow y \wedge z$, hence

$$
d_{\sigma}(x \wedge z, y \wedge z)=\lim d\left(x_{n} \wedge z_{n}, y_{n} \wedge z_{n}\right) \leq 2 \lim d\left(x_{n}, y_{n}\right)=2 d_{\sigma}(x, y) .
$$

Similarly, $d_{\sigma}(x \vee z, y \vee z) \leq 2 d_{\sigma}(x, y)$. Thus $d_{\sigma} \in D\left(S_{\sigma}\right)$.

By the definition of $d_{\sigma}$ and the exhaustivity of $d$, we have for $S \ni x_{n} \uparrow x$

$$
d_{\sigma}\left(x_{n}, x\right)=\lim _{j \rightarrow+\infty} d\left(x_{n}, x_{j}\right) \rightarrow 0(n \rightarrow+\infty),
$$

in particular, $S$ is lower dense in $S_{\sigma}$.

$d_{\sigma}$ is lower $\sigma$-o.c.: let now $S_{\sigma} \ni x_{n} \uparrow x$ and $\left(x_{n, i}\right)_{i}$ for each $n \in \mathbb{N}$ be a sequence in $S$ with $x_{n, i} \uparrow x_{n}$ (for $i \rightarrow+\infty$ ). Then $S \ni y_{n}:=\sup _{i, j \leq n} x_{i, j} \uparrow x$. Therefore $d_{\sigma}\left(y_{n}, x\right) \rightarrow$ $0(n \rightarrow+\infty)$ as we have seen before, hence $d_{\sigma}\left(x_{n}, x\right) \rightarrow 0(n \rightarrow+\infty)$ as $y_{n} \leq x_{n} \leq x$.

Since $S$ is dense in $\left(S_{\sigma}, d_{\sigma}\right)$ and $d$ is exhaustive, by [18, Proposition 6.14] $d_{\sigma}$ is exhaustive, too.

(b) Let $x \in S_{\sigma}$ and $\varepsilon>0$. By (a) there is an $y \in S$ with $y \leq x$ and $d_{\sigma}(x, y) \leq \varepsilon$. By the assumption in (b), there is a $z \in K$ with $z \leq y$ and $d_{\sigma}(y, z) \leq \varepsilon$. Therefore $x \geq z \in K$ and $d_{\sigma}(x, z) \leq 2 \varepsilon$.

(c) Let $x \in S_{\sigma}$ and $\varepsilon>0$. Let $x_{n} \in S$ with $x_{n} \uparrow x$ and $\left(z_{n}\right)$ an increasing sequence in $K$ with $d\left(x_{n}, z_{n}\right) \leq \varepsilon$ and $z_{n} \geq x_{n}$ for all $n \in \mathbb{N}$. (Such a sequence exists by the dual version of Lemma 2.3.) Then $x \leq z:=\sup z_{n} \in K_{\sigma}$ and $d_{\sigma}(x, z)=\lim d\left(x_{n}, z_{n}\right) \leq \varepsilon$.

By duality, we obtain from Proposition 4.1 the following result.

Proposition 4.2 Let $d \in D(S)$ be exhaustive and upper $\sigma$-o.c..

(a) Then there is a unique upper $\sigma$-o.c. $d_{\delta} \in D\left(S_{\delta}\right)$ extending $d$. Moreover, $S$ is upper dense in $\left(S_{\delta}, d_{\delta}\right)$, and $d_{\delta}$ is exhaustive.

(b) If $K$ is a upper-dense subset of $(S, d)$, then $K$ is upper dense in $\left(S_{\delta}, d_{\delta}\right)$, too. 
(c) If $K$ is a lower-dense subset of $(S, d)$, then $K_{\delta}$ is lower dense in $\left(S_{\delta}, d_{\delta}\right)$.

Remark 4.3 If in Proposition $4.1 d$ is $\sigma$-o.c., then $x_{n} \rightarrow x\left(d_{\sigma}\right)$ whenever $S_{\sigma} \ni x_{n} \downarrow x \in S$. On the other hand, in general $d_{\sigma}$ is not $\sigma$-o.c. as shown by Example 4.4.

Proof $S_{\sigma} \ni x_{n} \downarrow x \in S, \varepsilon>0$ and $\left(z_{n}\right)$ be a decreasing sequence in $S$ such that $z_{n} \leq x_{n}$ and $d\left(z_{n}, x_{n}\right) \leq \varepsilon$ for all $n \in \mathbb{N}$, see Lemma 2.3. Then $S \ni y_{n}:=x \vee z_{n} \downarrow x$, and thus $\lim d\left(y_{n}, x\right)=0$. With Lemma 2.2(b), we get

$$
d\left(x_{n}, x\right) \leq d\left(x_{n}, y_{n}\right)+d\left(y_{n}, x\right) \leq 2 d\left(x_{n}, z_{n}\right)+d\left(y_{n}, x\right) \leq 2 \varepsilon+d\left(y_{n}, x\right),
$$

and hence $d\left(x_{n}, x\right) \leq 3 \varepsilon$ eventually.

Example 4.4 Let $S:=[0,1[\cup] 2,3] \subseteq L:=[0,1] \cup] 2,3](\subseteq \mathbb{R})$ and $\bar{d}(x, y):=|x-y|$ for $x, y \in L$. Then $S_{\sigma}=L, \bar{d}$ is the unique lower $\sigma$-o.c. extension of $d:=\bar{d}_{\mid S \times S}$ belonging to $D(L), d$ is $\sigma$-o.c., but $\bar{d}$ is not.

$\bar{\mu}(x):=x$ defines a modular function on $L$. Let $\mu:=\bar{\mu}_{\mid S}$. Then the $\bar{\mu}$-uniformity ( $\mu$-uniformity) coincides with the $\bar{d}$-uniformity (respectively, with the $d$-uniformity). The function $\mu$ is $\sigma$-o.c., $\bar{\mu}$ is the unique lower $\sigma$-o.c. $\mathbb{R}$-valued modular extension of $\mu$, but $\bar{\mu}$ is not $\sigma$-o.c.: $2+\frac{1}{n} \downarrow 1$ in $L$, but $\mu\left(2+\frac{1}{n}\right) \nrightarrow \bar{\mu}(1)$.

Theorem 4.5 Let $d \in D(S)$ be exhaustive. Then the following conditions are equivalent:

(1) $\lim d\left(x_{n} \vee y_{n}, x_{n}\right)=0$ whenever $\left(x_{n}\right),\left(y_{n}\right)$ are sequences in $S$ and $x, y \in L$ such that $x_{n} \uparrow x, y_{n} \downarrow y$ and $y \leq x$.

(2) $d$ admits a $\sigma$-o.c. extension $d_{\sigma} \in D\left(S_{\sigma}\right)$ onto $S_{\sigma}$.

(3) d admits a $\sigma$-o.c. extension $\bar{d} \in D(\sigma(S))$ onto $\sigma(S)$.

Proof (3) $\Rightarrow$ (2) is obvious.

$(2) \Rightarrow(3)$ : First observe that by Proposition 4.1 the extension $d_{\sigma}$ is uniquely determined, $d_{\sigma}$ is exhaustive and $S$ is lower dense in $\left(S_{\sigma}, d_{\sigma}\right)$. By Proposition 4.2 applied to $S_{\sigma}, d_{\sigma}$ instead of $S, d$, there is a (unique) exhaustive upper $\sigma$-o.c. $\tilde{d} \in D\left(S_{\sigma \delta}\right)$ extending $d_{\sigma}$ such that $S_{\sigma}$ is upper dense in $\left(S_{\sigma \delta}, \tilde{d}\right)$. Moreover, 4.2(c) applied with $K:=S$ yields that $S_{\delta}$ is lower dense in $\left(S_{\sigma \delta}, \tilde{d}\right)$.

Let $\mathfrak{A}$ be the set of all pairs $\left(T, d_{T}\right)$ where $T$ is a sublattice of $\sigma(S)$ containing $S_{\sigma \delta}$, $d_{T} \in D(T)$ is an extension of $\tilde{d}, S_{\sigma}$ is upper dense in $\left(T, d_{T}\right)$ and $S_{\delta}$ is lower dense in $\left(T, d_{T}\right)$. As we have seen before $\left(S_{\sigma \delta}, \tilde{d}\right) \in \mathfrak{A}$. By Zorn's lemma, $\mathfrak{A}$ contains a maximal element $(M, \bar{d})$. Applying Lemma 2.6(a) for $S_{\delta}, M, \bar{d}$ instead of $K, M, d$, we get that $\bar{d}$ is upper $\sigma$-o.c., and applying Lemma 2.6(b) for $S_{\sigma}, M, \bar{d}$ instead of $K, M, d$, we get that $\bar{d}$ is lower $\sigma$-o.c.; thus $\bar{d}$ is $\sigma$-o.c. Since $S_{\sigma}$ is dense in $(M, \bar{d})$ and $d_{\sigma}=\bar{d}_{\mid S_{\sigma}}$ is exhaustive, $\bar{d}$ is by [18, Proposition 6.14] exhaustive, too.

It remains to show that $M=\sigma(S)$. We first show that $M_{\sigma}=M$ : by Proposition 4.1(a) there is a unique lower $\sigma$-o.c. $\hat{d} \in D\left(M_{\sigma}\right)$ extending $\bar{d}$. Moreover, applying 4.1(b) with $S_{\delta}, M$ instead of $K, S$ and 4.1(c) with $S_{\sigma}, M$ instead of $K, S$, we obtain that $S_{\delta}$ is lower dense and $S_{\sigma}$ is upper dense in $\left(M_{\sigma}, \hat{d}\right)$. Thus $\left(M_{\sigma}, \hat{d}\right) \in \mathfrak{A}$ and therefore $M_{\sigma}=M$ by the maximality of $M$. Similarly, one sees that $M_{\delta}=M$. Therefore $M=\sigma(S)$.

(2) $\Rightarrow(1)$ : With $x_{n}, y_{n}, x, y$ as in (1) we have

$$
\begin{aligned}
& d\left(x_{n} \vee y_{n}, x_{n}\right) \leq d_{\sigma}\left(x_{n} \vee y_{n}, x \vee y_{n}\right)+d_{\sigma}\left(x \vee y_{n}, x\right)+d_{\sigma}\left(x, x_{n}\right) \\
& \quad \leq 3 d_{\sigma}\left(x, x_{n}\right)+d_{\sigma}\left(x \vee y_{n}, x\right) .
\end{aligned}
$$

Now observe that $\lim d_{\sigma}\left(x, x_{n}\right)=\lim d_{\sigma}\left(x \vee y_{n}, x\right)=0$ since $x_{n} \uparrow x, x \vee y_{n} \downarrow x$ and $d_{\sigma}$ is $\sigma$-o.c.. 
(1) $\Rightarrow$ (2): Applying (1) with $y_{n}=y=x \in S$, one sees that $d$ is lower $\sigma$-o.c.. Let $d_{\sigma} \in D\left(S_{\sigma}\right)$ be the extension of $d$ according to Proposition 4.1. It remains to show that $d_{\sigma}$ is upper $\sigma$-o.c.: let $z_{n}, z \in S_{\sigma}$ with $z_{n} \downarrow z$ and $\varepsilon>0$. Choose a decreasing sequence $\left(y_{n}\right)$ in $S$ such that $y_{n} \leq z_{n}$ and $d_{\sigma}\left(y_{n}, z_{n}\right) \leq \varepsilon$ for all $n \in \mathbb{N}$ and an increasing sequence $\left(x_{n}\right)$ in $S$ with $x_{n} \uparrow z$. Then inf $y_{n} \leq \sup x_{n}$. Therefore $\lim d\left(x_{n} \vee y_{n}, x_{n}\right)=0$ by (1). From

$$
d_{\sigma}\left(z_{n}, z\right) \leq d_{\sigma}\left(z_{n}, x_{n} \vee y_{n}\right)+d_{\sigma}\left(x_{n} \vee y_{n}, x_{n}\right)+d_{\sigma}\left(x_{n}, z\right),
$$

$d_{\sigma}\left(z_{n}, x_{n} \vee y_{n}\right)=d_{\sigma}\left(x_{n} \vee z_{n}, x_{n} \vee y_{n}\right) \leq 2 d_{\sigma}\left(z_{n}, y_{n}\right) \leq 2 \varepsilon$ and $\lim d\left(x_{n} \vee y_{n}, x_{n}\right)=$ $\lim d_{\sigma}\left(x_{n}, z\right)=0$ it follows that $d_{\sigma}\left(z_{n}, z\right) \leq 3 \varepsilon$ eventually.

The next theorem is essentially a reformulation of Theorem 4.5.

Theorem 4.6 Let $u$ be an exhaustive lattice uniformity on $S$. Then the following conditions are equivalent:

(1) For any $U \in U$, we have $\left(x_{n} \vee y_{n}, x_{n}\right) \in U$ eventually whenever $\left(x_{n}\right),\left(y_{n}\right)$ are sequences in $S$ and $x, y \in L$ such that $x_{n} \uparrow x, y_{n} \downarrow y$ and $y \leq x$.

(2) There exists a $\sigma$-o.c. lattice uniformity $u_{\sigma}$ on $S_{\sigma}$ with $u_{\sigma \mid S}=u$.

(3) There exists a $\sigma$-o.c. lattice uniformity $\bar{u}$ on $\sigma(S)$ with $\bar{u}_{\mid S}=u$.

Proof (1) $\Rightarrow$ (3): Let $D$ be a subset of $D(S)$ generating $u$. Each $d \in D$ has by Theorem 4.5 (1) $\Rightarrow(3)$ a $\sigma$-o.c. extension $\bar{d} \in D(\sigma(S))$. Then the uniformity $\bar{u}$ generated by $\{\bar{d}: d \in D\}$ satisfies (3).

(3) $\Rightarrow$ (2) is obvious.

(2) $\Rightarrow(1)$ : Let $U \in u$ and $\bar{d} \in D\left(S_{\sigma}\right)$ be continuous with respect to $u_{\sigma}$ such that $\{(x, y) \in S: \bar{d}(x, y) \leq 1\} \subseteq U$. Now apply (2) $\Rightarrow$ (1) of Theorem 4.5 to $d:=\bar{d}_{\mid S \times S}$.

We now consider the case that $L$ is a complemented modular lattice or an orthomodular lattice. In this case, set $0=\min L$ and $1=\max L$.

Lemma 4.7 Let $L$ be a complemented modular lattice.

(a) If $a, b \in L$ with $a \leq b$ and $a^{\prime} \in L$ is a complement of $a$, then there is a complement $b^{\prime}$ of $b$ with $b^{\prime} \leq a^{\prime}$.

(b) If $z_{n} \uparrow z$ and $z_{n}^{\prime} \downarrow z^{\prime}$ in L such that $z_{n}^{\prime}$ is a complement of $z_{n}$ for each $n \in \mathbb{N}$, then $z^{\prime}$ is a complement of $z$.

Proof (a) By [6, Theorem I.14] $L$ is relatively complemented. One easily sees that a relative complement $b^{\prime}$ of $b \wedge a^{\prime}$ in $\left[0, a^{\prime}\right]$ is a complement of $b$ in $L$ with $b^{\prime} \leq a^{\prime}$.

(b) Since $L$ is $\sigma$-continuous, $1=z_{n} \vee z_{n}^{\prime} \leq z \vee z_{n}^{\prime} \downarrow z \vee z^{\prime}$ and hence $z \vee z^{\prime}=1$, and dually $0=z_{n} \wedge z_{n}^{\prime} \geq z_{n} \wedge z^{\prime} \uparrow z \wedge z^{\prime}$ and hence $z \wedge z^{\prime}=0$.

Corollary 4.8 Let $L$ be a complemented modular lattice and $S$ a sublattice of $L$ which contains all complements for any $x \in S$. Let $u$ be an exhaustive $\sigma$-o.c. lattice uniformity on $S$. Then there exists a unique $\sigma$-o.c. lattice uniformity $\bar{u}$ on $\sigma(S)$ with $\bar{u}_{\mid S}=u$.

Proof The uniqueness statement is contained in Theorem 3.1.

Existence: since $u$ is generated by a subset of $D(S)$, it is enough to consider the case that $u$ is generated by a single $d \in D(S)$. By Proposition 4.1, $d$ has a lower $\sigma$-o.c. extension $d_{\sigma} \in D\left(S_{\sigma}\right)$. By Theorem 4.5, it remains to show that $d_{\sigma}$ is upper $\sigma$-o.c.: let $z_{n}, z \in S_{\sigma}$ with $z_{n} \downarrow z$, and $\left(x_{n}\right)$ an increasing sequence in $S$ with $x_{n} \uparrow z$. By Lemma 4.7(a), there is a decreasing sequence $\left(x_{n}^{\prime}\right)$ in $S$ such that $x_{n}^{\prime}$ is a complement of $x_{n}$ for each $n \in \mathbb{N}$. By Lemma 
4.7 (b), $z^{\prime}:=\inf x_{n}^{\prime}$ is a complement of $z$. Consequently, $S_{\sigma} \ni z_{n} \wedge x_{n}^{\prime} \downarrow z \wedge z^{\prime}=0$ and therefore $d_{\sigma}\left(z_{n} \wedge x_{n}^{\prime}\right) \rightarrow 0$ by Remark 4.3. It follows, using $x_{n} \leq z \leq z_{n}$ and the modular law, that

$$
d_{\sigma}\left(z_{n}, z\right) \leq 2 d_{\sigma}\left(z_{n}, x_{n}\right)=2 d_{\sigma}\left(\left(z_{n} \wedge x_{n}^{\prime}\right) \vee x_{n}, 0 \vee x_{n}\right) \leq 4 d_{\sigma}\left(z_{n} \wedge x_{n}^{\prime}, 0\right) \rightarrow 0 .
$$

Corollary 4.9 Let $L=(L, \wedge, \vee, \perp)$ be an orthomodular lattice, $S$ a sub-orthomodular lattice of $L$ and $u$ an exhaustive $\sigma$-o.c. lattice uniformity on $S$. Then there exists a unique $\sigma$-o.c. lattice uniformity $\bar{u}$ on $\sigma(S)$ with $\bar{u}_{\mid S}=u$.

Proof The proof is almost identical with that of the proceeding corollary. One has to replace the complements $x_{n}^{\prime}$ by the orthocomplements $x_{n}^{\perp}$. By the rules in orthomodular lattices, we have $x_{n}^{\perp} \downarrow z^{\perp}$. Further observe that $\left(z_{n} \wedge x_{n}^{\perp}\right) \vee x_{n}=z_{n}$ by the orthomodular law.

As well known, the monotone system generated by an algebra of sets is a $\sigma$-algebra. B. Riečan generalized this fact in [13, Lemma on p. 240] and [14, Proposition 5]:

Remark 4.10 (B. Riečan)

(a) Under the assumption of Corollary 4.8, $\sigma(S)$ contains all complements for any $x \in \sigma(S)$.

(b) Under the assumptions of Corollary $4.9, \sigma(S)$ is a sub-orthomodular lattice.

\section{Extension of modular functions}

In this section, let $L$ be a $\sigma$-complete $\sigma$-continuous lattice, $S$ a sublattice of $L$ and $G$ be complete.

Theorem 5.1 Let $\mu: S \rightarrow G$ be an exhaustive modular function. Then the following conditions are equivalent:

(1) $\lim \mu\left(x_{n} \vee y_{n}\right)-\mu\left(x_{n}\right)=0$ whenever $\left(x_{n}\right),\left(y_{n}\right)$ are sequences in $S$ and $x, y \in L$ such that $x_{n} \uparrow x, y_{n} \downarrow y$ and $y \leq x$.

(2) There exists a $\sigma$-o.c. modular function $\mu_{\sigma}: S_{\sigma} \rightarrow G$ extending $\mu$.

(3) There exists a $\sigma$-o.c. modular function $\bar{\mu}: \sigma(S) \rightarrow G$ extending $\mu$.

Proof (3) $\Rightarrow$ (2) is obvious. (2) $\Rightarrow$ (3): By Proposition 2.5, the $\mu_{\sigma}$-uniformity $u_{\sigma}$ on $S_{\sigma}$ is $\sigma$-o.c.. Therefore by Theorem 4.6 (applied to $S_{\sigma}, u_{\sigma}$ instead of $S, u$ ), there exists a $\sigma$-o.c. lattice uniformity $\bar{u}$ on $\sigma(S)$ with $\bar{u}_{\mid S_{\sigma}}=u_{\sigma}$. By Theorem 3.1, $S$ is dense in $(\sigma(S), \bar{u})$. Therefore $\mu_{\sigma}$ admits a continuous extension $\bar{\mu}$ on $(\sigma(S), \bar{u})$ satisfying the condition (3).

(3) $\Rightarrow(1)$ : Let $x_{n}, y_{n}, x, y$ be given as in (1). Since the $\bar{\mu}$-uniformity $(=: \bar{u})$ is $\sigma$-o.c., we have $x_{n} \rightarrow x(\bar{u})$ and $y_{n} \rightarrow y(\bar{u})$, hence $x_{n} \vee y_{n} \rightarrow x \vee y=x(\bar{u})$. Consequently $\mu\left(x_{n} \vee y_{n}\right)-\mu\left(x_{n}\right) \rightarrow \bar{\mu}(x)-\bar{\mu}(x)=0(\tau)$.

(1) $\Rightarrow$ (2): Applying (1) with $y_{n}=y=x \in S$, one sees that $\mu$ is lower $\sigma$-o.c. Therefore the $\mu$-uniformity $u$ is lower $\sigma$-o.c. by Proposition 2.5. Let $\left(||_{\gamma}\right)_{\gamma \in \Gamma}$ be a system of bounded group seminorms generating $\tau$ and $\left(d_{\gamma}\right)_{\gamma \in \Gamma}$ the corresponding system of semimetrics on $S$ generating $u$ according to Proposition 2.4. By Proposition 4.1, each $d_{\gamma}$ has a lower $\sigma$-o.c. extension $\bar{d}_{\gamma} \in D\left(S_{\sigma}\right)$. Obviously, $S$ is lower dense in $S_{\sigma}$ with respect to the uniformity $\bar{u}$

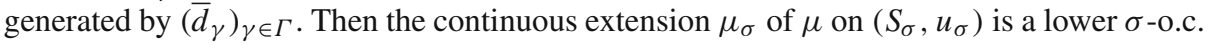
modular function. We proceed as in the proof of $4.5(1) \Rightarrow(2)$ to show that $\mu_{\sigma}$ is also upper 
$\sigma$-o.c.: let $z_{n}, z \in S_{\sigma}$ with $z_{n} \downarrow z$. Fix $\gamma \in \Gamma$ and $\varepsilon>0$. Choose a decreasing sequence $\left(y_{n}\right)$ in $S$ such that $y_{n} \leq z_{n}$ and $\bar{d}_{\gamma}\left(y_{n}, z_{n}\right) \leq \varepsilon$ for all $n \in \mathbb{N}$ and an increasing sequence $\left(x_{n}\right)$ in $S$ with $x_{n} \uparrow z$. Then inf $y_{n} \leq \sup x_{n}$. Therefore $\lim \mu\left(x_{n} \vee y_{n}\right)-\mu\left(x_{n}\right)=0$ by (1). From

$$
\begin{aligned}
\left|\mu_{\sigma}\left(z_{n}\right)-\mu_{\sigma}(z)\right|_{\gamma} \leq & \left|\mu_{\sigma}\left(z_{n}\right)-\mu_{\sigma}\left(x_{n} \vee y_{n}\right)\right|_{\gamma}+\left|\mu_{\sigma}\left(x_{n} \vee y_{n}\right)-\mu_{\sigma}\left(x_{n}\right)\right|_{\gamma} \\
& +\left|\mu_{\sigma}\left(x_{n}\right)-\mu_{\sigma}(z)\right|_{\gamma},
\end{aligned}
$$

$\left|\mu_{\sigma}\left(z_{n}\right)-\mu_{\sigma}\left(x_{n} \vee y_{n}\right)\right|_{\gamma} \leq \bar{d}_{\gamma}\left(x_{n} \vee z_{n}, x_{n} \vee y_{n}\right) \leq \bar{d}_{\gamma}\left(z_{n}, y_{n}\right) \leq \varepsilon$ and $\lim \mu\left(x_{n} \vee y_{n}\right)-$ $\mu\left(x_{n}\right)=\lim \mu_{\sigma}\left(x_{n}\right)-\mu_{\sigma}(z)=0$ follows that $\left|\mu_{\sigma}\left(z_{n}\right)-\mu_{\sigma}(z)\right|_{\gamma} \leq 2 \varepsilon$ eventually.

We have seen that $\lim \left|\mu_{\sigma}\left(z_{n}\right)-\mu_{\sigma}(z)\right|_{\gamma}=0$ for any $\gamma \in \Gamma$, i.e. $\mu_{\sigma}\left(z_{n}\right)-\mu_{\sigma}(z) \rightarrow$ $0(\tau)$.

Theorem 5.2 Let $L$ be a complemented modular lattice and $S$ a sublattice of $L$ which contains all complements for any $x \in S$.

Then for any $\sigma$-o.c. exhaustive modular function $\mu: S \rightarrow G$ there exists a unique $\sigma$-o.c. modular function $\bar{\mu}: \sigma(S) \rightarrow G$ extending $\mu$.

Proof The uniqueness statement is contained in Theorem 3.1.

Let $u$ be the $\mu$-uniformity and $\bar{u}$ its extension on $\sigma(S)$ according to Corollary 4.8. Then $S$ is dense in $(\sigma(S), \bar{u})$ by Theorem 3.1 and the continuous extension $\bar{\mu}$ of $\mu$ on $(\sigma(S), \bar{u})$ is $\sigma$-o.c. since $\bar{u}$ is $\sigma$-o.c.

Theorem 5.3 Let $L=(L, \wedge, \vee, \perp)$ be an orthomodular lattice and $S$ a sub-orthomodular lattice of $L$.

Then for any $\sigma$-o.c. exhaustive modular function $\mu: S \rightarrow G$ there exists a unique $\sigma$-o.c. modular function $\bar{\mu}: \sigma(S) \rightarrow G$ extending $\mu$.

Proof This can be proved as the proceeding theorem replacing Corollary 4.8 by Corollary 4.9 .

Remark 5.4 Let $M$ be an orthomodular lattice or a modular complemented lattice. Then $\mu: M \rightarrow G$ is additive (i.e. $\mu(x \vee y)=\mu(x)+\mu(y)$ whenever $x, y \in M$ and $x \wedge y=0$ ) iff $\mu$ is a modular function with $\mu(0)=0$.

Proof The implication $\Leftarrow$ is obvious. $\Rightarrow$ : In case of $M$ being an orthomodular lattice see [20, Proposition 3.1]. If $M$ is modular and complemented, one proceeds similarly: let $x, y \in M$. Since $M$ is relatively complemented, $x \wedge y$ has a relative complement $z$ in $[0, x]$. One easily sees that then $z$ is a relative complement of $y$ in $[0, x \vee y]$. Therefore

$\mu(x \vee y)+\mu(x \wedge y)=(\mu(y)+\mu(z))+\mu(x \wedge y)=\mu(y)+(\mu(z)+\mu(x \wedge y))=\mu(y)+\mu(x)$.

\section{Extension of D-uniformities and modular measures on D-lattices and pseudo-D-lattices}

\subsection{D-lattices}

In this section, let $L$ be a $\sigma$-complete $\sigma$-continuous $D$-lattice, $S$ a $D$-sublattice of $L$ and $G$ be complete.

Recall that a $D$-lattice $L=(L, \wedge, \vee, \ominus)$ is a lattice with a smallest element 0 , a greatest element 1 and a partial operation $\ominus$ on $L$ such that $b \ominus a$ is defined iff $a \leq b$ and for all $a, b, c \in L$ the following two rules are satisfied: 
If $a \leq b$ then $b \ominus a \leq b$ and $b \ominus(b \ominus a)=a$,

if $a \leq b \leq c$ then $c \ominus b \leq c \ominus a$ and $(c \ominus a) \ominus(c \ominus b)=b \ominus a$.

A $D$-sublattice of $L$ is a sublattice closed with respect to $\ominus$ and containing 1. Examples for D-lattices are MV-algebras and orthomodular lattices. An orthomodular lattice becomes a D-lattice with $b \ominus a:=b \wedge a^{\perp}$ if $a \leq b$.

A $D$-uniformity on $L$ is a lattice uniformity on $L$ such that $\ominus$ is uniformly continuous, or equivalently, that $\triangle: L \times L \rightarrow L$ defined by $a \Delta b:=(a \vee b) \ominus(a \wedge b)$ is uniformly continuous. Let $D_{0}(L)$ be the set of all $d \in D(L)$ such that $d(x \Delta z, y \Delta z) \leq 2 d(x, y)$. It follows from [19, Proposition 1.2] that the D-uniformities on $L$ are exactly the uniformities generated by a nonempty subset of $D_{0}(L)$.

We need the following estimation:

Lemma 6.1 $d(a, b)=d(b \triangle(b \ominus a), b \triangle 0) \leq 2 d(b \ominus a, 0)$ if $d \in D_{0}(L)$ and $a \leq b$.

A modular measure $\mu: L \rightarrow G$ is a modular function such that $\mu(b \ominus a)=\mu(b)-\mu(a)$ whenever $a, b \in L$ and $a \leq b$. If $\mu$ is a modular measure, then the $\mu$-uniformity is always a D-uniformity, see [1, 4.2]. D-uniformities and modular measures were intensively studied by A. Avallone and P. Vitolo. In a series of articles, they (partly with coauthors) transferred various theorems for measures on Boolean algebras to the setting of modular measures on D-lattices. Hereby, the D-uniformities play a similar role as FN-topologies in the study of measures on Boolean algebras.

If an orthomodular lattice is considered as D-lattice, then any lattice uniformity is a Duniformity. This follows from the fact that a lattice uniformity on an orthomodular lattice also makes the orthocomplementation $x \mapsto x^{\perp}$ uniformly continuous, see [20, Theorem 1.1]. Moreover, a modular function $\mu$ on an orthomodular lattice is a modular measure iff $\mu(0)=0$.

The following lemma is essentially contained in [7, Section 1.8] and in this formulation in [3, Lemma 3.2].

Lemma 6.2 Let $x_{n}, y_{n}, x, y \in L$ for $n \in \mathbb{N}$. Then

$y \geq x_{n} \uparrow x$ implies $y \ominus x_{n} \downarrow y \ominus x$, and $y \geq x_{n} \downarrow x$ implies $y \ominus x_{n} \uparrow y \ominus x$;

$x \leq y_{n} \uparrow y$ implies $y_{n} \ominus x \uparrow y \ominus x$, and $x \leq y_{n} \downarrow y$ implies $y_{n} \ominus x \downarrow y \ominus x$

Lemma 6.3 (a) If $S \ni x \leq y \in S_{\sigma}$, then $y \ominus x \in S_{\sigma}$.

(b) If $M$ is a sublattice of $L$, then $M_{\sigma} \triangle M_{\sigma} \subseteq(M \triangle M)_{\sigma \delta}$ and $M_{\delta} \triangle M_{\delta} \subseteq(M \triangle M)_{\sigma \delta}$.

Proof (a) If $S \ni y_{n} \uparrow y$, then $S \ni x \vee y_{n} \uparrow y$ and hence $S \ni\left(x \vee y_{n}\right) \ominus x \uparrow y \ominus x$ by Lemma 6.2.

(b) Set $A=M \triangle M$. Let $x, y \in M_{\sigma}$ and $x_{n}, y_{n} \in M$ with $x_{n} \uparrow x$ and $y_{n} \uparrow y$. For fixed $k \in \mathbb{N}$, we have by Lemma 6.2

$$
A \ni\left(x_{k+n} \vee y_{k+n}\right) \ominus\left(x_{k} \wedge y_{k}\right) \uparrow(x \vee y) \ominus\left(x_{k} \wedge y_{k}\right) \in A_{\sigma},
$$

and therefore $A_{\sigma} \ni(x \vee y) \ominus\left(x_{n} \wedge y_{n}\right) \downarrow(x \vee y) \ominus(x \wedge y) \in A_{\sigma \delta}$. This proves $M_{\sigma} \triangle M_{\sigma} \subseteq A_{\sigma \delta}$.

Similarly, if $M \ni x_{n} \downarrow x$ and $M \ni y_{n} \downarrow y$, then

$$
A \ni\left(x_{k} \vee y_{k}\right) \ominus\left(x_{k+n} \wedge y_{k+n}\right) \uparrow\left(x_{k} \vee y_{k}\right) \ominus(x \wedge y) \in A_{\sigma},
$$

therefore $A_{\sigma} \ni\left(x_{n} \vee y_{n}\right) \ominus(x \wedge y) \downarrow(x \vee y) \ominus(x \wedge y) \in A_{\sigma \delta}$. This proves $M_{\delta} \triangle M_{\delta} \subseteq A_{\sigma \delta}$.

The following result was first proved in [3, Theorem 3.4]. Here we give an essentially easier proof, which could be of interest also in the Boolean case. 
Theorem $6.4 \sigma(S)$ is a D-sublattice of $L$.

Proof Let $\mathfrak{A}$ be the set of all sublattices $T$ of $\sigma(S)$ containing $S$ such that $T \triangle T \subseteq \sigma(S)$. Then $S \in \mathfrak{A}$. Let $M$ be a maximal element of $\mathfrak{A}$. By Lemma 6.3, we have $M_{\sigma} \triangle M_{\sigma} \subseteq$ $(M \triangle M)_{\sigma \delta} \subseteq \sigma(S)_{\sigma \delta}=\sigma(S)$, hence $M_{\sigma} \in \mathfrak{A}$, and thus $M_{\sigma}=M$ by the maximality of $M$. Similarly, one sees $M_{\delta}=M$. Therefore $\sigma(M)=M$ and finally $M=\sigma(S)$, since $S \subseteq M \subseteq \sigma(S)$.

Theorem 6.5 Let $d \in D_{0}(S)$ be exhaustive and $\sigma$-o.c.. Then $d$ has a unique $\sigma$-o.c. extension $\bar{d} \in D_{0}(\sigma(S))$.

Proof The uniqueness statement is contained in Theorem 3.1.

Existence: Let $d_{\sigma} \in D\left(S_{\sigma}\right)$ be according to Proposition 4.1. We first show that $d_{\sigma}$ is upper $\sigma$-o.c. As in the proof of Corollary 4.8, let $z_{n}, z \in S_{\sigma}$ with $z_{n} \downarrow z$ and $\left(x_{n}\right)$ an increasing sequence in $S$ with $x_{n} \uparrow z$. Then $z_{n} \ominus x_{n} \in S_{\sigma}$ by Lemma 6.3(a) and $z_{n} \ominus x_{n} \downarrow 0$ by Lemma 6.2. Hence $d_{\sigma}\left(z_{n} \ominus x_{n}, 0\right) \rightarrow 0$ by Remark 4.3. Therefore, using $x_{n} \leq z \leq z_{n}$ and Lemma 6.1, we have $d_{\sigma}\left(z_{n}, z\right) \leq 2 d_{\sigma}\left(z_{n}, x_{n}\right) \leq 4 d_{\sigma}\left(z_{n} \ominus x_{n}, 0\right) \rightarrow 0$.

By Theorem $4.5 d$ has a $\sigma$-o.c. extension $\bar{d} \in D(\sigma(S))$. It remains to show that $\bar{d}(x \triangle$ $z, y \Delta z) \leq 2 \bar{d}(x, y)$ for all $x, y, z \in \sigma(S)$. Let $\mathfrak{A}$ be the set of all sublattices $T$ of $\sigma(S)$ containing $S$ such that $\bar{d}(x \triangle z, y \Delta z) \leq 2 \bar{d}(x, y)$ for all $x, y, z \in T$. By Zorn's lemma, $\mathfrak{A}$ contains a maximal element $M$. Let $x, y, z \in M_{\sigma}$ and $x_{n}, y_{n}, z_{n} \in M$ such that $x_{n} \uparrow x$, $y_{n} \uparrow y$ and $z_{n} \uparrow z$. We show that $x_{n} \Delta z_{n} \rightarrow x \Delta z(\bar{d})$. Since $x_{i}, z_{i} \in M \in \mathfrak{A}$, we have

$$
\bar{d}\left(\left(x_{k} \vee z_{k}\right) \triangle\left(x_{n} \wedge z_{n}\right),\left(x_{n} \vee z_{n}\right) \Delta\left(x_{n} \wedge z_{n}\right)\right) \leq 2 \bar{d}\left(x_{k} \vee z_{k}, x_{n} \vee z_{n}\right) .
$$

Passing to the limit for a fixed $n \in \mathbb{N}$ and $n<k \rightarrow+\infty$ (see the previous formula), we get

$$
\bar{d}\left((x \vee z) \triangle\left(x_{n} \wedge z_{n}\right), x_{n} \triangle z_{n}\right) \leq 2 \bar{d}\left(x \vee z, x_{n} \vee z_{n}\right) \text {. }
$$

Therefore

$$
\begin{aligned}
\bar{d}\left(x \Delta z, x_{n} \triangle z_{n}\right) & \leq \bar{d}\left(x \Delta z,(x \vee z) \Delta\left(x_{n} \wedge z_{n}\right)\right)+\bar{d}\left((x \vee z) \Delta\left(x_{n} \wedge z_{n}\right), x_{n} \triangle z_{n}\right) \\
& \leq \bar{d}\left(x \Delta z,(x \vee z) \triangle\left(x_{n} \wedge z_{n}\right)\right)+2 \bar{d}\left(x \vee z, x_{n} \vee z_{n}\right) .
\end{aligned}
$$

Since $(x \vee z) \triangle\left(x_{n} \wedge z_{n}\right) \downarrow x \Delta z, x_{n} \vee z_{n} \uparrow x \vee z$ and $\bar{d}$ is $\sigma$-o.c., we have

$$
\lim \bar{d}\left(x \triangle z,(x \vee z) \triangle\left(x_{n} \wedge z_{n}\right)\right)=\lim \bar{d}\left(x \vee z, x_{n} \vee z_{n}\right)=0
$$

and hence $\lim \bar{d}\left(x \triangle z, x_{n} \triangle z_{n}\right)=0$.

As we have seen, $x_{n} \Delta z_{n} \rightarrow x \Delta z(\bar{d})$ and analogously $y_{n} \Delta z_{n} \rightarrow y \Delta z(\bar{d})$; moreover, $x_{n} \rightarrow x(\bar{d})$ and $y_{n} \rightarrow y(\bar{d})$. Therefore from $\bar{d}\left(x_{n} \triangle z_{n}, y_{n} \Delta z_{n}\right) \leq 2 \bar{d}\left(x_{n}, y_{n}\right)$ we obtain passing to the limit $\bar{d}(x \Delta z, y \triangle z) \leq 2 \bar{d}(x, y)$.

This proves that $M_{\sigma} \in \mathfrak{A}$, and by the maximality of $M$ that $M_{\sigma}=M$.

Similarly, one can prove that $M_{\delta}=M$, starting with the inequality

$$
\bar{d}\left(\left(x_{n} \vee z_{n}\right) \triangle\left(x_{k} \wedge z_{k}\right),\left(x_{n} \vee z_{n}\right) \Delta\left(x_{n} \wedge z_{n}\right)\right) \leq 2 \bar{d}\left(x_{k} \wedge z_{k}, x_{n} \wedge z_{n}\right)
$$

where $x_{k}, z_{k} \in M$ with $x_{k} \downarrow x$ and $z_{k} \downarrow z$.

Finally, $M_{\sigma}=M_{\delta}=M$ implies $M=\sigma(S)$, i.e. $\bar{d} \in D_{0}(\sigma(S))$.

Corollary 6.6 Let $u$ be an exhaustive $\sigma$-o.c. D-uniformity on $S$. Then there exists a unique $\sigma$-o.c. D-uniformity $\bar{u}$ on $\sigma(S)$ with $\bar{u}_{\mid S}=u$.

This immediately follows from Proposition 3.1, Theorem 6.5 and the fact that any Duniformity on $S$ or $\sigma(S)$ is generated by semimetrics of $D_{0}(S)$ or $D_{0}(\sigma(S))$, respectively. 
Corollary 6.7 Let $\mu: S \rightarrow G$ be a $\sigma$-o.c. exhaustive modular measure. Then there exists a unique $\sigma$-o.c. modular measure $\bar{\mu}: \sigma(S) \rightarrow G$ extending $\mu$.

Proof We proceed as in the proofs of 5.2 and 5.3. Let $u$ be the $\mu$-uniformity and $\bar{u}$ its extension on $\sigma(S)$ according to Corollary 6.6. Then $S$ is dense in $(\sigma(S), \bar{u})$ by Theorem 3.1. Define $\bar{\mu}$ as the continuous extension of $\mu$ on $(\sigma(S), \bar{u})$. The uniqueness statement is contained in Theorem 3.1.

Corollary 6.6 generalizes Corollary 4.9. Corollary 6.7 generalizes the extension theorem for measures on orthomodular lattices (Theorem 5.3) as well as for measures on MV-algebras [5, Theorem 3.2.2].

\subsection{Pseudo-D-lattices}

In this section, let $L$ be a $\sigma$-complete $\sigma$-continuous pseudo-D-lattice, $S$ a $D$-sublattice of $L$ and $G$ be complete.

Pseudo-D-lattices (= lattice ordered pseudoeffect algebras) were introduced by Dvurečenskij and Vetterlein [8] as "noncommutative version" of D-lattices. For the definition and basic properties, we refer to [8] and [4]. On $L$ are defined besides the lattice operations $\vee, \wedge$ two partial binary operations $\backslash$ and / which can be extended on $L \times L$ by

$$
a^{*} \triangle b=(a \vee b) \backslash(a \wedge b) \text { and } a \triangle^{*} b=(a \wedge b) /(a \vee b) \text {. }
$$

If $L$ is a D-lattice, then ${ }^{*} \triangle$ and $\Delta^{*}$ coincide with $\triangle$.

Let us recall the definition of a D-uniformity. This is a lattice uniformity on $L$ making \ and / uniformly continuous, or equivalently, a lattice uniformity making ${ }^{*} \triangle$ and $\triangle^{*}$ uniformly continuous. Let $D_{0}(L)$ be the set of all $d \in D(L)$ such that $d\left(x^{*} \triangle z, y^{*} \triangle z\right) \leq 2 d(x, y)$ and $d\left(x \Delta^{*} z, y \Delta^{*} z\right) \leq 2 d(x, y)$. By [19, Proposition 1.2], the D-uniformities on $L$ are exactly the uniformities generated by a nonempty subset of $D_{0}(L)$.

As 6.1 and 6.3 one can verify the following facts using the usual rules of calculation in pseudo-D-lattices, in particular the identity $(b \backslash a) / b=a$ for $a \leq b$ and rules for the two "differences" \and / analogous to 6.2.

Lemma 6.8 (a) $d(a, b) \leq 2 d(b \backslash a, 0)$ if $d \in D_{0}(L)$ and $a \leq b$.

(b) If $S \ni x \leq y \in S_{\sigma}$, then $y \backslash x \in S_{\sigma}$.

(c) If $M$ is a sublattice of $L$, then $\left(M_{\sigma}{ }^{*} \triangle M_{\sigma}\right) \cup\left(M_{\delta}{ }^{*} \triangle M_{\delta}\right) \subseteq\left(M^{*} \triangle M\right)_{\sigma \delta}$ and $\left(M_{\sigma} \triangle^{*}\right.$ $\left.M_{\sigma}\right) \cup\left(M_{\delta} \Delta^{*} M_{\delta}\right) \subseteq\left(M \Delta^{*} M\right)_{\sigma \delta}$.

The next theorem, which coincides with [4, Theorem 4.2], can be proved in the same way as Theorem 6.4 using Lemma 6.8 (c) instead of 6.3(b). This leads to a proof simpler than that given in [4].

Theorem $6.9 \sigma(S)$ is a D-sublattice of $L$.

It is near at hand how to modify proofs of Sect. 6.1 to obtain the following results.

Theorem 6.10 (a) Any $\sigma$-o.c. exhaustive $d \in D_{0}(S)$ has a unique $\sigma$-o.c. extension $\bar{d} \in$ $D_{0}(\sigma(S))$.

(b) Any $\sigma$-o.c. exhaustive D-uniformity $u$ on $S$ has a unique $\sigma$-o.c. D-uniformity $\bar{u}$ on $\sigma(S)$ with $\bar{u}_{\mid S}=u$.

Corollary 6.11 Let $\mu: S \rightarrow G$ be a $\sigma$-o.c. exhaustive modular measure. Then there exists a unique $\sigma$-o.c. modular measure $\bar{\mu}: \sigma(S) \rightarrow G$ extending $\mu$. 
Such an extension theorem was proved in [4, Corollaries 5.5 and 5.6] under the additional assumption that $G$ is a locally convex linear space, or that $G$ is a normed group and $\mu$ has finite variation.

\section{References}

1. Avallone, A., Basile, A.: On a Marinacci uniqueness theorem for measures. J. Math. Anal. Appl. 286, 378-390 (2003)

2. Avallone, A., De Simone, A.: Extensions of modular functions on orthomodular lattices. Ital. J. Pure Appl. Math. 9, 109-122 (2001)

3. Avallone, A., De Simone, A., Vitolo, P.: Effect algebras and extensions of measures. Bollettino U.M.I. 9-B(8), 423-444 (2006)

4. Avallone, A., De Simone, A., Vitolo, P.: Extension of measures on pseudo-D-lattices. Math. Slovaca 66, 421-438 (2016)

5. Barbieri, G., Weber, H.: A topological approach to the study of fuzzy measures. In: Abramovich, Y., Avgerinos, E., Yannelis, N.C. (eds.) Functional Analysis and Economic Theory (Samos, 1996), pp. 1746. Springer, Berlin (1998)

6. Birkhoff, G.: Lattice Theory, 3rd edn. American Mathematical Society, Providence (1984)

7. Dvurečenskij, A., Pulmannová, S.: New Trends in Quantum Structures. Mathematics and Its Applications, vol. 516. Kluwer Academic Publishers, Norwell (2000)

8. Dvurečenskij, A., Vetterlein, T.: Pseudoeffect algebras. I. Basic properties. Int. J. Theor. Phys. 40, 685-701 (2001)

9. Fleischer, I., Traynor, T.: Group-valued modular functions. Algebra Univ. 14, 287-291 (1982)

10. Fox, G., Morales, P.: Strongly additive function on lattices. Fundam. Math. 78, 99-106 (1973)

11. Kranz, P.: Extension of a valuation on a lattice. Fundam. Math. 91, 171-178 (1976)

12. Riečan, B.: On the extension of a measure on lattices. Mat. Časopis Sloven. Akad. Vied 19, 44-49 (1969)

13. Riečan, B.: A note on the extension of measures on lattices. Mat. Časopis Sloven. Akad. Vied 20, 239-244 (1970). [Zbl 0205.07104 (Autorreferat in Zentralblatt für Mathematik)]

14. Riečan, B.: The measure extension theorem for subadditive probability measures in orthomodular $\sigma$-continuous lattices. Comment. Math. Univ. Carolin. 20, 309-316 (1979)

15. Riečanová, Z.: Topology in a quantum logic induced by a measure. In: Proceedings of the Conference: Topology and Measure, V (Binz, 1987), pp. 126-130, Greifswald (1988)

16. Sion, M.: Outer measures with values in a topological group. Proc. Lond. Math. Soc. 19, 89-106 (1969)

17. Weber, H.: Uniform lattices I: a generalization of topological Riesz spaces and topological Boolean rings. Ann. Mat. Pura Appl. 160, 347-370 (1991)

18. Weber, H.: Uniform lattices II: order continuity and exhaustivity. Ann. Mat. Pura Appl. 165, 133-158 (1993)

19. Weber, H.: Metrization of uniform lattices. Czechoslovak Math. J. 43(118), 271-280 (1993)

20. Weber, H.: Lattice uniformities and modular functions on orthomodular lattices. Order 12, 295-305 (1995)

21. Weber, H.: On modular functions. Funct. Approx. 24, 35-52 (1996) 